\title{
Prediction of Dissociation Constant in Protein-Ligand Interaction
}

\author{
Masatoshi Kawashima \\ Laboratory Section, Azuma Co., Ltd. \\ 1-6-13 Tamasaki-Nishi, Ichihara, Chiba 290-0044 \\ E-mail: kawashima@azuma-g.co.jp
}

In protein-ligand interactions, such as antigen-antibody interactions and hormone-receptor interactions, a correlation between the equilibrium dissociation constant $K_{\mathrm{D}}$ and the reduced mass of the protein and ligand was found. The correlation of dissociation constants as $\mathrm{p} K_{\mathrm{D}}\left(-\log K_{\mathrm{D}}\right)$ between literature values and predicted values was confirmed in high coefficient of determination $\mathrm{R}^{2}$ over 0.98 .

\section{Keywords: Dissociation Constant |Protein-Ligand Interaction |Correlation |Reduced Mass |Prediction}

The author has reported the correlation between reduced mass and yield in many organic chemical reactions, such as $\mathrm{C}$ $\mathrm{C}, \mathrm{C}-\mathrm{N}, \mathrm{C}-\mathrm{O}$ coupling reactions, cycloaddition reactions, Bingel reaction of fullerene, and general reactions in the synthesis of natural products and has shown that the relationship expressed in the following equations 1a-e for the reaction scheme 1 is maintained even in enzymatic reactions including formation of ES complex..$^{1-5}$ Therefore, the author

$$
\mathrm{A}+\mathrm{B} \longrightarrow \mathrm{C}(+\mathrm{D})
$$

Scheme 1. Reaction of molecule A with B to produce C (and D)

$$
\begin{aligned}
& y=-0.186 \frac{M_{\mathrm{AB}}^{\prime}}{n_{\mathrm{A}} n_{\mathrm{B}} n_{\mathrm{I}}}+100 \\
& M_{\mathrm{AB}}^{\prime}=\frac{M_{\mathrm{A}}^{\prime} M_{\mathrm{B}}^{\prime}}{M_{\mathrm{A}}^{\prime}+M_{\mathrm{B}}^{\prime}} \\
& M_{\mathrm{A}(\mathrm{B})}^{\prime}=M_{\mathrm{A}(\mathrm{B})}-14.03 a R_{\mathrm{A}(\mathrm{B})} \\
& a=0.00177 M_{\mathrm{A}(\mathrm{B})} \quad\left(M_{\mathrm{A}(\mathrm{B})} \leq 768\right) \\
& a=1.36 \quad\left(M_{\mathrm{A}(\mathrm{B})}>768\right)
\end{aligned}
$$

$$
y \quad \text { : yield of molecule C }
$$

$M_{\mathrm{AB}}^{\prime}$ : adjusted reduced mass of molecular A and B

$M_{\mathrm{A}(\mathrm{B})}^{\prime}$ : adjusted molecular weight of molecular A(B)

$M_{\mathrm{A}(\mathrm{B})}$ : molecular weight of molecular A(B)

$n_{\mathrm{A}(\mathrm{B})}:$ number of reaction sites $\mathrm{A}(\mathrm{B})$

$n_{\mathrm{I}} \quad:$ intermolecular reaction $=1$, intramolecular or enzymatic reaction $=2$

$R_{\mathrm{A}(\mathrm{B})}:$ number of rotatable bonds of molecular $\mathrm{A}(\mathrm{B})$

$a$ : coefficient for adjustment by molecular weight was interested in the relationship between reduced mass and protein-ligand interactions, such as antigen-antibody reactions and hormone-receptor interactions. For these protein-ligand interactions, it was considered appropriate to replace the yield, which is a measure of the correlation with reduced mass used in the analysis of organic reactions reported previously, with the equilibrium dissociation constant $K_{\mathrm{D}}$. The $K_{D}$, which represents binding affinity, is very important in studying protein-ligand interactions, and methods for measuring, evaluating, and predicting $K_{\mathrm{D}}$ are considered important. ${ }^{6}$ In this report, we would like to show a simple $K_{\mathrm{D}}$ prediction method using reduced mass, which has not been published before.

As for antigen-antibody reactions, the author analyzed the reactions between antigens, that cause malignant tumors, allergies, and autoimmune diseases, and their antibody drugs, as well as the reactions between proteins on the outer membrane of SARS-CoV-2 virus that causes the new coronavirus infection (COVID-19), and receptors on the cell membranes of infected cells. For drug-receptor interactions, the reactions with receptors for insulin, insulin-like growth factor, calcitonin, calcitriol, and thyroid hormone were analyzed. In an equilibrium reaction shown in scheme 2 , the dissociation constant $K_{\mathrm{D}}$ is expressed by the equation $2 \mathrm{a}$. Since the yield $y$ is expressed in the equation $2 b$, if the concentrations of $\mathrm{A}$ and $\mathrm{B}$ are equal and the initial concentration of molecule $\mathrm{A}$ is $[\mathrm{A}]_{0} \mathrm{M}$, the equation relating $K_{\mathrm{D}}$ and $y$ can be obtained and is shown in the equation $2 \mathrm{~d}$. The

$$
[\mathrm{AB}] \rightleftharpoons[\mathrm{A}]+[\mathrm{B}]
$$

Scheme 2. Equilibrium reaction: $\mathrm{AB} \rightleftarrows \mathrm{A}+\mathrm{B}$

$$
\begin{aligned}
& K_{D}=\frac{[\mathrm{A}][\mathrm{B}]}{[\mathrm{AB}]} \\
& y=100 \frac{[\mathrm{AB}]}{[\mathrm{A}]_{0}}=100 \frac{[\mathrm{A}]_{0}-[\mathrm{A}]}{[\mathrm{A}]_{0}} \\
& {[\mathrm{~A}]=[\mathrm{B}]} \\
& K_{\mathrm{D}}=[\mathrm{A}]_{0} \frac{(100-y)^{2}}{100 y}
\end{aligned}
$$

$K_{\mathrm{D}}$ was calculated by substituting $y$, which was obtained using the equation $1 \mathrm{a}$, into the equation $2 \mathrm{~d}$. The number of rotatable bonds (NORB) of a protein was determined by first dividing the molecular weight by the average mass of amino acid 
residues $\left(\frac{M_{\mathrm{A}(\mathrm{B})}}{118.8}\right)$, multiplying it by the average number of rotatable bonds of amino acids (2.90), and then subtracting the number of amide bonds $\left(\frac{M_{\mathrm{A}(\mathrm{B})}}{118.8}\right)$ from it as follows: ${ }^{7}$

$$
R_{\mathrm{A}(\mathrm{B})}=2.90 \frac{M_{\mathrm{A}(\mathrm{B})}}{118.8}-\frac{M_{\mathrm{A}(\mathrm{B})}}{118.8}=0.01599 M_{\mathrm{A}(\mathrm{B})} .
$$

In the previous report on the correlation between reduced mass and yield in enzymatic reactions, it was suggested that $n_{\mathrm{I}}$ in the equation $1 \mathrm{a}$ represents affinity. Therefore, $n_{\mathrm{I}}$ was expressed as the equation 3 using the number of binding sites or ligands $\left(n_{\mathrm{A}}, n_{\mathrm{B}}\right)$, and the coefficient $c$ was adjusted to optimal value in each concentration $[\mathrm{A}]_{0}$ so that the regression coefficient of the relationship between the literature and predicted value of $\mathrm{p} K_{\mathrm{D}}\left(\mathrm{p} K_{\mathrm{D}}=-\log K_{\mathrm{D}}\right)$ calculated by using the rewritten equation $1 \mathrm{a}^{\prime}$ and the equation $2 \mathrm{~d}$, in the graph would be closer to 1 .

$$
\begin{aligned}
& n_{I}=c n_{A} n_{B} \\
\therefore \quad & y=-\frac{0.186 M_{A B}^{\prime}}{c\left(n_{A} n_{B}\right)^{2}}+100
\end{aligned}
$$

Table 1 summarizes protein-ligand combinations analyzed (sample size $=64$ ), molecular weight, number of rotatable bonds, reduced mass adjusted with NORB, $n_{\mathrm{A}}, n_{\mathrm{B}}, n$ $\left(=c\left(n_{\mathrm{A}} n_{\mathrm{B}}\right)^{2}\right)$, and the literature values of dissociation constant and their predictions in the case of $[\mathrm{A}]_{0}=1 \times 10^{-6} \mathrm{M} \cdot{ }^{8-42}$ In the table, the literature values of $K_{\mathrm{D}}$ were listed without considering the differences in measurement methods and conditions such as temperature, $\mathrm{pH}$, and salt concentration. Literature versus predicted dissociation constants $\mathrm{p} K_{\mathrm{D}}$ in the cases of protein concentration of $1 \mathrm{M}, 1 \times 10^{-2} \mathrm{M}, 1 \times 10^{-4} \mathrm{M}$, and $1 \times 10^{-6} \mathrm{M}$ are shown in Figure 1 . (a)

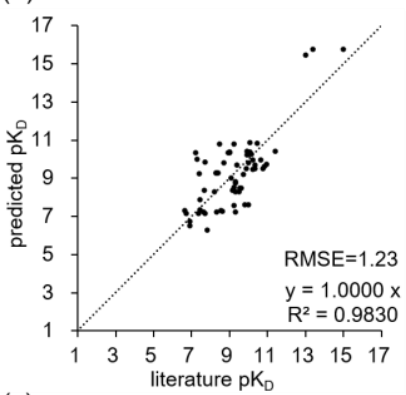

(c)

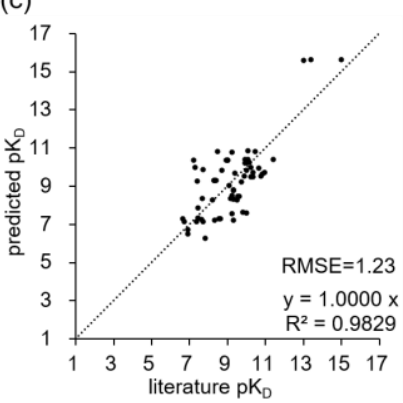

(b)

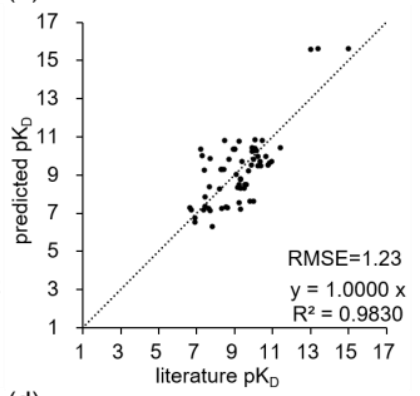

(d)

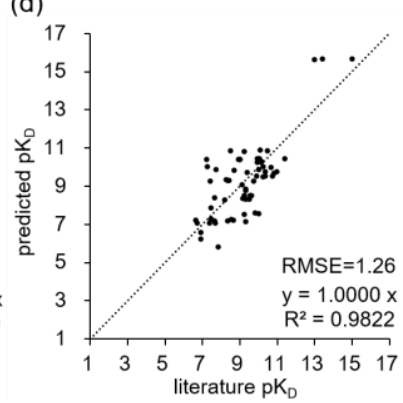

Figure 1. Literature versus predicted $\mathrm{p} K_{\mathrm{D}}$ plot of proteinligand interaction. (a) $[\mathrm{A}]_{0}=1 \mathrm{M}$, (b) $[\mathrm{A}]_{0}=1 \times 10^{-2} \mathrm{M}$, (c) $[\mathrm{A}]_{0}=1 \times 10^{-4} \mathrm{M}$, (d) $[\mathrm{A}]_{0}=1 \times 10^{-6} \mathrm{M}$.

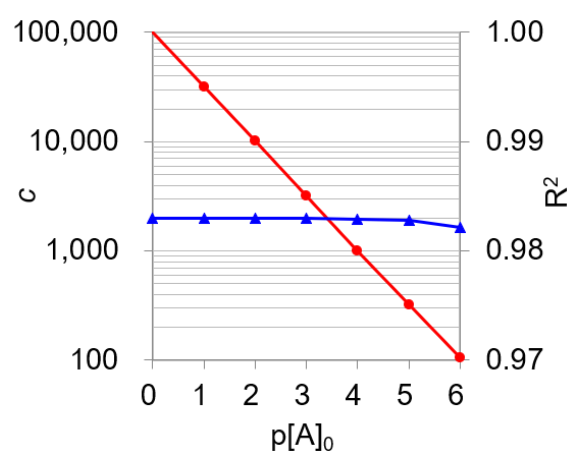

Figure 2. $c$ value $(\bullet)$ for each $[\mathrm{A}]_{0}$ and coefficient of determination $\mathrm{R}^{2}(\mathbf{\Delta})$ in the correlation between literature and predicted $\mathrm{p} K_{\mathrm{D}}$.

As shown in Figure 2, the $c$ value refers to the collision frequency, and the relationship with $[\mathrm{A}]_{0}$ is expressed by the equation:

$$
c=99882[\mathrm{~A}]_{0}^{0.4973} \quad\left(\mathrm{R}^{2}=1.00\right)
$$

Coefficient of determination $\mathrm{R}^{2}$ in the correlation between the literature and predicted $\mathrm{p} K_{\mathrm{D}}$ is over 0.98 in a wide range of $[\mathrm{A}]_{0}$. Although this method does not consider the structure or free energy at all, a higher coefficient of determination was obtained than the prediction method by machine learning using X-ray crystal resolution. ${ }^{6(b, c)}$ Furthermore, this $K_{\mathrm{D}}$ prediction method can also be used to estimate the number of binding sites by changing $n_{\mathrm{A}}$ and $n_{\mathrm{B}}$ to see if it agrees with the predicted value, if the exact measurement of $K_{\mathrm{D}}$ is known.

This method was applied to the prediction of dissociation constant of an insect pheromone and the receptor. The dissociation constants for the pheromone (+)-disparlure of the gypsy moth Lymantria dispar and the pheromone binding proteins PBP2 and DNS-PB2 are $2.9 \times 10^{-6} \mathrm{M}$ and $2.6 \times 10^{-6} \mathrm{M}$, respectively. ${ }^{43}$ To make the predictions from equation $1 \mathrm{a}^{\prime}$ and $2 \mathrm{~d}$ agree with these literature values, $c$ had to be lowered to 0.43 (predicted values of $2.8 \times 10^{-6} \mathrm{M}$ and $2.9 \times 10^{-6}$ ), which is much smaller than the value (106) calculated as $[\mathrm{A}]_{0}=1 \times 10^{-6}$ $M$ using the equation 4. According to the literature, this interaction takes place in two steps at two different sites of the receptor, which may be one of the reasons. 
Table 1. Analysis of protein-ligand interaction in the case of $[\mathrm{A}]_{0}=1 \times 10^{-6} \mathrm{M}$.

\begin{tabular}{|c|c|c|c|c|c|c|c|c|c|c|c|c|c|c|}
\hline & & & & & & & & & & & & $K_{\mathrm{D}}$ & (M) & \\
\hline A & B & $M_{\mathrm{A}^{\mathrm{a}}}$ & ref. $^{b}$ & $M_{\mathrm{B}}^{\mathrm{c}}$ & ref. ${ }^{d}$ & $R_{\mathrm{A}}{ }^{e}$ & $R_{\mathrm{B}}^{\mathrm{f}}$ & $M_{\mathrm{AB}^{\mathrm{g}}}$ & $n_{\mathrm{A}}{ }^{\mathrm{h}}$ & $n_{\mathrm{B}}^{\mathrm{i}}$ & $n^{\mathrm{j}}$ & literature ${ }^{k}$ & predicted & ref. ${ }^{1}$ \\
\hline $\begin{array}{l}\text { Melanotransferrin } \\
\text { (mTF) }\end{array}$ & anti $\mathrm{mTF} s \mathrm{scF}$ & 80215 & 44 & 28000 & 8 & 1283 & 448 & 14421 & 1 & 1 & 106 & $2.04 \times 10^{-8}$ & $8.7 \times 10^{-8}$ & 8 \\
\hline $\mathrm{mTF}$ & anti mTF IgG & 80215 & & 150000 & 8 & 1283 & 2399 & 36316 & 2 & 2 & 1688 & $4.82 \times 10^{-10}$ & $1.7 \times 10^{-9}$ & 8 \\
\hline $\begin{array}{l}\text { CD20, B-lymphocyte } \\
\text { antigen }\end{array}$ & $\begin{array}{l}\text { murine anti-CD20 } \\
\mathrm{mAb} 2 \mathrm{~B} 8\end{array}$ & 35000 & 9 & 150000 & 51 & 560 & 2399 & 19718 & 2 & 2 & 1688 & $3.88 \times 10^{-9}$ & $4.8 \times 10^{-10}$ & 9 \\
\hline $\mathrm{CD} 20$ & rituximab & 35000 & & 144187 & 46 & 560 & 2306 & 19569 & 2 & 2 & 1688 & $5.45 \times 10^{-9}$ & $4.8 \times 10^{-10}$ & 9 \\
\hline CD20 & ofatumumab & 35000 & & 146060 & 46 & 560 & 2336 & 19618 & 2 & 2 & 1688 & $4.76 \times 10^{-9}$ & $4.8 \times 10^{-10}$ & 9 \\
\hline $\begin{array}{l}\text { human epidermal } \\
\text { growth factor } \\
\text { receptor } 2 \text { (HER2) }\end{array}$ & trastuzumab & 137910 & 44 & 145532 & 47 & 2206 & 2328 & 49201 & 2 & 4 & 6752 & $4 \times 10^{-10}$ & $1.9 \times 10^{-10}$ & 10 \\
\hline HER2 & pertuzumab & 137910 & & 148000 & 48 & 2206 & 2367 & 49603 & 2 & 2 & 1688 & $3 \times 10^{-10}$ & $3.2 \times 10^{-9}$ & 10 \\
\hline $\begin{array}{l}\text { Vascular endothelial } \\
\text { growth factor } \\
\text { (VEGF)-A165 }\end{array}$ & aflibercept & 45000 & 44 & 96897 & 47 & 720 & 1550 & 21352 & 2 & 2 & 1688 & $1.9 \times 10^{-10}$ & $5.7 \times 10^{-10}$ & 12 \\
\hline VEGF-A165 & bevacizumab & 27042 & & 149197 & 47 & 432 & 2386 & 15907 & 2 & 2 & 1688 & $5.8 \times 10^{-11}$ & $3.1 \times 10^{-10}$ & 12 \\
\hline VEGF-A165 & ranibizumab & 27042 & & 48379 & 47 & 432 & 774 & 12053 & 2 & 2 & 1688 & $4.6 \times 10^{-11}$ & $1.8 \times 10^{-10}$ & 12 \\
\hline $\begin{array}{l}\text { epidermal growth } \\
\text { factor receptor (EGFR) }\end{array}$ & cetuximab & 134277 & 44 & 152000 & 49 & 2148 & 2431 & 49538 & 2 & 2 & 1688 & $2.5 \times 10^{-10}$ & $3.2 \times 10^{-9}$ & 13 \\
\hline EGFR & imgatuzumab (GA201) & 134277 & & 145027 & 47 & 2148 & 2319 & 48446 & 2 & 2 & 1688 & $6 \times 10^{-10}$ & $3.0 \times 10^{-9}$ & 11 \\
\hline $\begin{array}{l}\text { hen egg white } \\
\text { lysozyme (HEL) }\end{array}$ & $\begin{array}{l}\text { hen egg white lysozyme } \\
\text { antibody (HyHEL-10) }\end{array}$ & 14305 & 14 & 150000 & 14 & 229 & 2399 & 9074 & 2 & 2 & 1688 & $2.2 \times 10^{-11}$ & $1.0 \times 10^{-11}$ & 14 \\
\hline $\begin{array}{l}\text { cluster of } \\
\text { differentiation } 4 \text { (CD4) }\end{array}$ & ibalizumab & 51111 & 44 & 150000 & 50 & 817 & 2399 & 26488 & 2 & 2 & 1688 & $8 \times 10^{-10}$ & $8.8 \times 10^{-10}$ & 11 \\
\hline $\begin{array}{l}\text { surface antigen CD319 } \\
\text { (SLAMF7) }\end{array}$ & elotuzumab & 37421 & 44 & 145452 & 46 & 598 & 2326 & 20681 & 2 & 2 & 1688 & $4.04 \times 10^{-8}$ & $5.3 \times 10^{-10}$ & 15 \\
\hline $\begin{array}{l}\text { tumor necrosis factor } \\
(\mathrm{TNF} \alpha)\end{array}$ & golimumab & 25644 & 44 & 146943 & 46 & 410 & 2350 & 15171 & 2 & 2 & 1688 & $1.8 \times 10^{-11}$ & $2.8 \times 10^{-10}$ & 16 \\
\hline $\mathrm{TNF} \alpha$ & etanercept & 25644 & & 51167 & 46 & 410 & 818 & 11870 & 2 & 2 & 1688 & $1.1 \times 10^{-11}$ & $1.7 \times 10^{-10}$ & 16 \\
\hline $\mathrm{TNF} \alpha$ & infliximab & 25644 & & 165000 & 56 & 410 & 2639 & 15422 & 2 & 2 & 1688 & $4.4 \times 10^{-11}$ & $2.9 \times 10^{-10}$ & 16 \\
\hline $\mathrm{TNF} \alpha$ & adalimumab & 25644 & & 150000 & 56 & 410 & 2399 & 15217 & 2 & 2 & 1688 & $1.3 \times 10^{-10}$ & $2.9 \times 10^{-10}$ & 16 \\
\hline $\begin{array}{l}\text { complement } \\
\text { component } 5 \text { (C5) }\end{array}$ & eculizumab & 188305 & 44 & 148000 & 46 & 3012 & 2367 & 57580 & 2 & 2 & 1688 & $6.9 \times 10^{-10}$ & $4.3 \times 10^{-9}$ & 17 \\
\hline C5 & ravulizumab & 188305 & & 144937 & 46 & 3012 & 2318 & 56907 & 2 & 2 & 1688 & $2.2 \times 10^{-8}$ & $4.2 \times 10^{-9}$ & 17 \\
\hline SARS-CoV-2 S & $\begin{array}{l}\text { angiotensin-converting } \\
\text { enzyme } 2 \text { (ACE2) }\end{array}$ & 141178 & 44 & 92463 & 44 & 2258 & 1479 & 38821 & 1 & 1 & 106 & $1.47 \times 10^{-8}$ & $1.5 \times 10^{-6}$ & 18 \\
\hline $\mathrm{V}-2 \mathrm{~S} 1$ & ACE2 & 78300 & 57 & 92463 & & 1252 & 1479 & 29459 & 1 & 1 & 106 & $1.2 \times 10^{-7}$ & $5.6 \times 10^{-7}$ & 19 \\
\hline SARS-CoV-2 S RBD & $\mathrm{ACE} 2$ & 51500 & 57 & 92463 & & 824 & 1479 & 22983 & 1 & 1 & 106 & $1.2 \times 10^{-7}$ & $2.8 \times 10^{-7}$ & 19 \\
\hline SARS-CoV-2 RBD & $\mathrm{ACE} 2$ & 23400 & 52 & 92463 & & 374 & 1479 & 12975 & 1 & 1 & 106 & $4.7 \times 10^{-9}$ & $6.8 \times 10^{-8}$ & 20 \\
\hline $\begin{array}{l}\text { SARS-Cov-2-RBD- } \\
\text { His tag }\end{array}$ & $\mathrm{ACE} 2$ & 26000 & 53 & 92463 & & 416 & 1479 & 14101 & 1 & 1 & 106 & $4.42 \times 10^{-8}$ & $8.2 \times 10^{-8}$ & 20 \\
\hline $\begin{array}{l}\text { SARS-Cov-RBD- } \\
\text { His tag }\end{array}$ & $\mathrm{ACE} 2$ & 26500 & 54 & 92463 & & 424 & 1479 & 14311 & 1 & 1 & 106 & $1.85 \times 10^{-7}$ & $8.5 \times 10^{-8}$ & 20 \\
\hline SARS-CoV-2 RBD & humanized sdAbs 1E2 & 23400 & 55 & 15000 & 21 & 374 & 240 & 6351 & 1 & 1 & 106 & $3.552 \times 10^{-8}$ & $1.4 \times 10^{-8}$ & 21 \\
\hline $\begin{array}{l}\text { PIP HOE } 140, \\
\text { a radioligand }\end{array}$ & bradykinin B2 receptor & 1381 & 45 & 44461 & 44 & 28 & 711 & 824 & 1 & 1 & 106 & $1.5 \times 10^{-11}$ & $2.1 \times 10^{-10}$ & 22 \\
\hline $\begin{array}{l}\text { WAY-100635, } \\
\text { an antagonist }\end{array}$ & 5-HT(1A) receptor & 423 & 45 & 46107 & 44 & 7 & 737 & 345 & 1 & 1 & 106 & $8.7 \times 10^{-11}$ & $3.7 \times 10^{-11}$ & 23 \\
\hline $\begin{array}{l}{\left[{ }^{3} \mathrm{H}\right] \mathrm{CP} 96345,} \\
\text { a tritium-labeled } \\
\text { antagonist }\end{array}$ & NK1 receptor & 413 & 45 & 46251 & 44 & 7 & 740 & 337 & 1 & 1 & 106 & $1.2 \times 10^{-10}$ & $3.6 \times 10^{-11}$ & 24 \\
\hline $\begin{array}{l}\text { Olmesartan, an } \\
\text { antagonist }\end{array}$ & $\begin{array}{l}\text { Human angiotensin II } \\
\text { AT1 receptor }\end{array}$ & 559 & 45 & 41061 & 44 & 11 & 657 & 400 & 1 & 1 & 106 & $9.1 \times 10^{-11}$ & $5.0 \times 10^{-11}$ & 25 \\
\hline $\begin{array}{l}\text { Telmisartan, an } \\
\text { antagonist }\end{array}$ & $\begin{array}{l}\text { Human angiotensin II } \\
\text { AT1 receptor }\end{array}$ & 515 & 45 & 41061 & & 7 & 657 & 419 & 1 & 1 & 106 & $1.2 \times 10^{-10}$ & $5.5 \times 10^{-11}$ & 25 \\
\hline insulin & insulin receptor (IR) & 5808 & 45 & 156333 & 44 & 93 & 2500 & 3891 & 1 & 1 & 106 & $6.41 \times 10^{-9}$ & $5.1 \times 10^{-9}$ & 26 \\
\hline $\begin{array}{l}\text { Insulin-like growth } \\
\text { factor } 1 \text { (IGF-1) }\end{array}$ & $\begin{array}{l}\text { insulin-like growth } \\
\text { factor } 1 \text { receptor (IGF- } \\
\text { IR) }\end{array}$ & 21841 & 45 & 154793 & 44 & 349 & 2476 & 13299 & 1 & 1 & 106 & $5 \times 10^{-10}$ & $7.2 \times 10^{-8}$ & 27 \\
\hline $\begin{array}{l}\text { Insulin-like growth } \\
\text { factor } 2 \text { (IGF-2) }\end{array}$ & IGF-1R & 20140 & 44 & 154793 & & 322 & 2476 & 12383 & 1 & 1 & 106 & $2.3 \times 10^{-9}$ & $6.1 \times 10^{-8}$ & 28 \\
\hline insulin & $\begin{array}{l}\text { insulin receptor } \\
\text { A (IR-A) }\end{array}$ & 5808 & & 125000 & 58 & 93 & 1999 & 3856 & 1 & 1 & 106 & $3.2 \times 10^{-10}$ & $5.0 \times 10^{-9}$ & 28 \\
\hline IGF-1 & IR-A & 21841 & & 125000 & & 349 & 1999 & 12919 & 1 & 1 & 106 & $2.37 \times 10^{-8}$ & $6.7 \times 10^{-8}$ & 28 \\
\hline IGF-2 & IR-A & 20140 & & 125000 & & 322 & 1999 & 12052 & 1 & 1 & 106 & $2.9 \times 10^{-9}$ & $5.7 \times 10^{-8}$ & 28 \\
\hline
\end{tabular}

${ }^{\mathrm{a}}$ molecular weight of molecule $\mathrm{A},{ }^{\mathrm{b}, \mathrm{d}}$ reference for molecular weight of molecule $\mathrm{A}(\mathrm{B}){ }^{\mathrm{c}}$ molecular weight of molecule $\mathrm{B},{ }^{\mathrm{e}}$ number of rotatable bonds of molecule $\mathrm{A},{ }^{\mathrm{f}}$ number of rotatable bonds of molecule $\mathrm{B},{ }^{\mathrm{g}}$ reduced mass adjusted with NORB $\left(R_{\mathrm{A}}, R_{\mathrm{B}}\right),{ }^{\mathrm{h}}$ number of bonding sites or number of ligands (molecule A), ${ }^{i}$ number of bonding sites or number of ligands (molecule B), h,i In the case of antigen/monoclonal antibody interaction, $n_{\mathrm{A}}$ and $n_{\mathrm{B}}$ were both set to 2 unless otherwise stated in the literature, ${ }^{\mathrm{j}} n=c\left(n_{\mathrm{A}} n_{\mathrm{B}}\right)^{2}$, $c=105.5,{ }^{\mathrm{k}}$ If there was a range of literature values, the value with the higher affinity (smaller $K_{\mathrm{D}}$ value) was listed. ${ }^{1}$ reference for $K_{\mathrm{D}}$. 
Table 1. continued

\begin{tabular}{|c|c|c|c|c|c|c|c|c|c|c|c|c|c|c|}
\hline & & & & & & & & & & & & $K_{\mathrm{D}}($ & & \\
\hline A & B & $M_{\mathrm{A}}{ }^{\mathrm{a}}$ & ref. ${ }^{b}$ & $M_{\mathrm{B}}^{\mathrm{c}}$ & ref. ${ }^{d}$ & $R_{\mathrm{A}} \mathrm{e}^{\mathrm{s}}$ & $R_{\mathrm{B}}^{\mathrm{f}}$ & $M_{\mathrm{AR}}^{\mathrm{g}}$ & $n_{A}{ }^{h}$ & $n_{\mathrm{B}}^{\mathrm{i}}$ & $n n^{\mathrm{j}}$ & literature $^{k}$ & predicted & ref. ${ }^{1}$ \\
\hline insulin & $\begin{array}{l}\text { insulin receptor isoform } \\
\text { B (IR-B) }\end{array}$ & 5808 & & 90000 & 58 & 93 & 1439 & 3791 & 1 & 1 & 106 & $5 \times 10^{-10}$ & $4.8 \times 10^{-9}$ & 28 \\
\hline IGF-1 & IR-B & 21841 & & 90000 & & 349 & 1439 & 12212 & 1 & 1 & 106 & $2.24 \times 10^{-7}$ & $5.9 \times 10^{-8}$ & 28 \\
\hline IGF-2 & IR-B & 20140 & & 90000 & & 322 & 1439 & 11435 & 1 & 1 & 106 & $3.55 \times 10^{-8}$ & $5.1 \times 10^{-8}$ & 28 \\
\hline IGF-1 & $\begin{array}{l}\text { Insulin-like growth } \\
\text { factor-binding protein } 1 \\
\text { (IGFBP-1) } \\
\text { insulin-like growth }\end{array}$ & 21841 & & 27904 & 44 & 349 & 446 & 8513 & 1 & 1 & 106 & $1 \times 10^{-10}$ & $2.7 \times 10^{-8}$ & 29 \\
\hline IGF-1 & $\begin{array}{l}\text { factor-binding protein } 3 \\
\text { (IGFBP-3) }\end{array}$ & 21841 & & 31674 & 44 & 349 & 507 & 8982 & 1 & 1 & 106 & $6 \times 10^{-10}$ & $3.0 \times 10^{-8}$ & 30 \\
\hline $\begin{array}{l}\text { calcitonin (chicken), } \\
\text { a peptide hormone }\end{array}$ & $\begin{array}{l}\text { chicken calcitonin } \\
\text { receptor }\end{array}$ & 3372 & 45 & 32423 & 59 & 54 & 519 & 2122 & 1 & 1 & 106 & $5 \times 10^{-10}$ & $1.5 \times 10^{-9}$ & 31 \\
\hline calcitonin & $\begin{array}{l}\text { calcitonin receptors } \\
\text { in rat osteoclasts }\end{array}$ & 15103 & 44 & 60292 & 44 & 242 & 964 & 8392 & 1 & 1 & 106 & $1.6 \times 10^{-10}$ & $2.6 \times 10^{-8}$ & 32 \\
\hline $\begin{array}{l}\text { calcitriol, } \\
\text { the active form of } \\
\text { vitamin D }\end{array}$ & $\begin{array}{l}\text { endoplasmic reticulum } \\
\text { resident protein } 57 \\
\text { (ERp57) }\end{array}$ & 417 & 45 & 56782 & 44 & 5 & 908 & 362 & 1 & 1 & 106 & $1 \times 10^{-9}$ & $4.1 \times 10^{-11}$ & 33 \\
\hline calcitriol & $\begin{array}{l}\text { vitamin } \mathrm{D} \text { receptor } \\
\text { (VDR) }\end{array}$ & 417 & & 48289 & 44 & 5 & 772 & 361 & 1 & 1 & 106 & $1.2 \times 10^{-9}$ & $4.1 \times 10^{-11}$ & 34 \\
\hline calcitriol & $\begin{array}{l}\text { caveolae-enriched } \\
\text { membrane fraction } \\
\text { (CMF) }\end{array}$ & 417 & & 23000 & 60 & 5 & 368 & 357 & 1 & 1 & 106 & $1 \times 10^{-9}$ & $4.0 \times 10^{-11}$ & 34 \\
\hline calcitriol & $\begin{array}{l}\text { plasma vitamin } \mathrm{D} \\
\text { binding protein (DBP) }\end{array}$ & 417 & & 58000 & 61 & 5 & 928 & 362 & 1 & 1 & 106 & $6 \times 10^{-8}$ & $4.1 \times 10^{-11}$ & 34 \\
\hline $\begin{array}{l}\text { AMG580, } \\
\text { a PET tracer }\end{array}$ & $\begin{array}{l}\text { phosphodiesterase 10A } \\
\text { (PED10A) }\end{array}$ & 473 & 35 & 88412 & 44 & 6 & 1414 & 400 & 1 & 1 & 106 & $7.19 \times 10^{-11}$ & $5.0 \times 10^{-11}$ & 35 \\
\hline $\begin{array}{l}\text { propranolol, } \\
\text { a beta blocker }\end{array}$ & $\beta$-1-adrenergic receptor & 259 & 45 & 51323 & 44 & 7 & 821 & 213 & 1 & 1 & 106 & $3.3 \times 10^{-9}$ & $1.4 \times 10^{-11}$ & 36 \\
\hline propranolol & $\beta$-2-adrenergic receptor & 259 & 45 & 46459 & 44 & 6 & 743 & 219 & 1 & 1 & 106 & $6.0 \times 10^{-10}$ & $1.5 \times 10^{-11}$ & 36 \\
\hline $\begin{array}{l}{\left[{ }^{3} \mathrm{H}\right]-\text { dihydroalprenolol }} \\
\text { (DHA), a trithium- } \\
\text { labeled blocker } \\
{\left[{ }^{125} \mathrm{I}\right]-}\end{array}$ & $\beta$-2-adrenergic receptor & 251 & 45 & 46459 & & 8 & 743 & 200 & 1 & 1 & 106 & $8.35 \times 10^{-11}$ & $1.2 \times 10^{-11}$ & 37 \\
\hline $\begin{array}{l}\text { iodocyanopindolol } \\
\text { (CYP), a radiolabeled } \\
\text { antagonist }\end{array}$ & $\beta$-2-adrenergic receptor & 399 & 45 & 46459 & & 6 & 743 & 336 & 1 & 1 & 106 & $4.1 \times 10^{-12}$ & $3.5 \times 10^{-11}$ & 37 \\
\hline $\begin{array}{l}\text { triiodothyronine (T3), } \\
\text { a thyroid hormone }\end{array}$ & $\begin{array}{l}\text { thyroid hormone } \\
\text { receptor } \alpha 1 \text { (THR } \alpha 1)\end{array}$ & 651 & 45 & 54816 & 44 & 5 & 877 & 562 & 1 & 1 & 106 & $6 \times 10^{-11}$ & $9.9 \times 10^{-11}$ & 38 \\
\hline $\begin{array}{l}\text { thyroxine (T4), } \\
\text { a thyroid hormone }\end{array}$ & $\mathrm{THR} \alpha 1$ & 777 & 45 & 54816 & & 5 & 877 & 669 & 1 & 1 & 106 & $2 \times 10^{-9}$ & $1.4 \times 10^{-10}$ & 38 \\
\hline T4 & $\begin{array}{l}\text { thyroxinebinding } \\
\text { globulin (TBG) }\end{array}$ & 777 & & 46325 & 44 & 5 & 741 & 667 & 1 & 1 & 106 & $1 \times 10^{-10}$ & $1.4 \times 10^{-10}$ & 39 \\
\hline $\mathrm{T} 3$ & transthyretin(TTR) & 651 & & 15887 & 44 & 5 & 254 & 542 & 1 & 1 & 106 & $5.326 \times 10^{-8}$ & $9.2 \times 10^{-11}$ & 40 \\
\hline $\mathrm{T} 4$ & TTR & 777 & & 15887 & & 5 & 254 & 642 & 1 & 1 & 106 & $1.973 \times 10^{-8}$ & $1.3 \times 10^{-10}$ & 40 \\
\hline biotin, vitamin B7 & $\begin{array}{l}\text { avidin, a biotin-binding } \\
\text { protein }\end{array}$ & 244 & 45 & 54700 & 62 & 5 & 875 & 213 & 4 & 4 & 27008 & $1 \times 10^{-15}$ & $2.1 \times 10^{-16}$ & 41 \\
\hline thiobiotin & avidin & 260 & 45 & 54700 & & 5 & 875 & 227 & 4 & 4 & 27008 & $1 \times 10^{-13}$ & $2.4 \times 10^{-16}$ & 41 \\
\hline iminobiotin & avidin & 243 & 45 & 54700 & & 5 & 875 & 212 & 1 & 1 & 106 & $3.5 \times 10^{-11}$ & $1.4 \times 10^{-11}$ & 41 \\
\hline biotin & streptavidin & 244 & & 53361 & 63 & 5 & 853 & 213 & 4 & 4 & 27008 & $4 \times 10^{-14}$ & $2.1 \times 10^{-16}$ & 42 \\
\hline
\end{tabular}

${ }^{\mathrm{a}}$ molecular weight of molecule $\mathrm{A},{ }^{\mathrm{b}, \mathrm{d}}$ reference for molecular weight of molecule A(B) ${ }^{\mathrm{c}}$ molecular weight of molecule $\mathrm{B},{ }^{\mathrm{e}}$ number of rotatable bonds of molecule $\mathrm{A},{ }^{\mathrm{f}}$ number of rotatable bonds of molecule $\mathrm{B},{ }^{\mathrm{g}}$ reduced mass adjusted with $\mathrm{NORB}\left(R_{\mathrm{A}}, R_{\mathrm{B}}\right),{ }^{\mathrm{h}}$ number of bonding sites or number of ligands (molecule A), ${ }^{\mathrm{i}}$ number of bonding sites or number of ligands (molecule B), h,i In the case of antigen/monoclonal antibody interaction, $n_{\mathrm{A}}$ and $n_{\mathrm{B}}$ were both set to 2 unless otherwise stated in the literature, ${ }^{\mathrm{j}} n=c\left(n_{\mathrm{A}} n_{\mathrm{B}}\right)^{2}$, $c=105.5,{ }^{\mathrm{k}}$ If there was a range of literature values, the value with the higher affinity (smaller $K_{\mathrm{D}}$ value) was listed. ${ }^{1}$ reference for $K_{\mathrm{D}}$.

\section{References and Notes}

1 M. Kawashima, ChemRxiv. 2020, Preprint. https://doi.org/10.26434/chemrxiv.12993218.v1
2 M. Kawashima, ChemRxiv. 2020, Preprint. https://doi.org/10.26434/chemrxiv.13350974.v1

3 M. Kawashima, ChemRxiv. 2020, Preprint. https://doi.org/10.26434/chemrxiv.13465718.v1

4 M. Kawashima, ChemRxiv. 2021, Preprint. https://doi.org/10.26434/chemrxiv.13536080.v1 
5 M. Kawashima, ChemRxiv. 2021, Preprint. https://doi.org/10.26434/chemrxiv.13670737.v1

6 (a) I. Jarmoskaite, I. AlSadhan, P. P Vaidyanathan, D. Herschlag, eLife 2020, 9, e57264. (b) R. Reverberi, L. Reverberi, Blood Transfus. 2007, 5, 227. (c) R. Dias, B. Kolaczkowski, BMC Bioinform. 2017, 18, 102.

7 D. F. Veber, S. R. Johnson, H.-Y. Cheng, B. R. Smith, K. W. Ward, K. D. Kopple, J. Med. Chem. 2002, 45, 2615.

8 R. Sato, T. Obonai, R. Tsumura, K. Tsumoto, Y. Koga, M. Yasunaga, Y. Matsumura, Cancer Sci. 2014, 105, 1631.

9 S. Uchiyama, Y. Suzuki, K. Otake, M. Yokoyama, M. Ohta, S. Aikawa, M. Komatsu, T. Sawada, Y. Kagami, Y. Morishima, K. Fukui, Cancer Sci. 2010, 101, 201.

10 M. W. Pedersen, H. J. Jacobsen, K. Koefoed, A. Dahlman, I. Kjær, T. T. Poulsen, P.-J. Meijer, L. S. Nielsen, I. D. Horak, J. Lantto, M. Kragh, Mol. Cancer Ther. 2015, 14, 669.

11 Y. Mazor, C. Yang, M. J. Borrok, J. Ayriss, K. Aherne, H. Wu, W. F. Dall'Acqua, PLoS One 2016, 11, e0157788.

12 G. Sumner, C. Georgaros, A. Rafique, T. DiCioccio, J. Martin, N. Papadopoulos, T. Daly, A. Torri, Bioanalysis 2019, 11, 381.

13 M. Eiblmaier, L. A. Meyer, M. A. Watson, P. M. Fracasso, L. J. Pike, C. J. Anderson, J. Nucl. Med. 2008, 49, 1472.

14 K. A. Xavier, R. C. Willson, Biophys. J. 1998, 74, 2036.

15 A. Ghai, J. Nucl. Med. 2020, 61, 1068.

16 D. Shealy, A. Cai, K. Staquet, A. Baker, E. R Lacy, L. Johns, O. Vafa, G. Gunn III, S. Tam, S. Sague, D. Wang, M. Brigham-Burke, P. Dalmonte, E. Emmell, B. Pikounis, P. J Bugelski, H. Zhou, B. Scallon, J. Giles-Komar, MAbs. 2010, 2, 428.

17 D. Sheridan, Z.-X. Yu, Y. Zhang, R. Patel, F. Sun, M. A. Lasaro, K. Bouchard, B. Andrien, A. Marozsan, Y. Wang, P. Tamburini, PLoS One. 2018; 13, 0195909.

18 Y. Huang, C. Yang, X.-F. Xu, W. Xu1, S.-W. Liu, Acta Pharmacol. Sin. 2020, 41, 1141.

19 J. Yang, S. J. L. Petitjean, M. Koehler, Q. Zhang, A. C. Dumitru, W. Chen, S. Derclaye, S. P. Vincent, P. Soumillion, D. Alsteens, Nat. Commun. 2020, 11, 4541.

20 J. Shang, G. Ye, K. Shi, Y. Wan, C. Luo, H. Aihara, Q. Geng, A. Auerbach, F. Li, Nature 2020, 581, 221.

21 X. Chi, X. Liu, C. Wang, X. Zhang, X. Li, J. Hou, L. Ren, Q. Jin, J. Wang, W. Yang, Nat. Commun. 2020, 11, 4528.

22 N. J. Brenner, G. Y. Stonesifer, K. A. Schneck, H. D. Burns, R. W. Ransom, Life Sci. 1993, 53, 1879.

23 S. Parkel, A. Rinken, Neurochem. Res. 2006, 31, 1135.

24 X.-L. Zhang. J. C. W. Mak, P. J. Barnes, Peptides 1995, 16, 867.

25 M. T. Le, M. K. Pugsley, G Vauquelin, I V. Liefde, Br. J. Pharmacol. 2007, 151, 952.

26 K. Subramaniana, C. J. Feea, R. Fredericksa, R. S. Stubbsb, M. T. Hayesb, J. Mol. Recognit. 2013, 26, 643 .

27 M. Keyhanfar, G. W. Booker, J. Whittaker, J. C. Wallace, B. E. Forbes, Biochem. J. 2007, 401, 269.

28 K. Macháčková, M. Chrudinová, J. Radosavljević, P. Potalitsyn, K. Kř́žková, M. Fábry, I. Selicharová, M. Collinsová, A. M. Brzozowski, L. Žáková, J. Jiráček, Biochemistry 2018, 57, 2373.

29 D. Tian, P. K. Kreeger, BMC Syst. Biol. 2014, 8, 98.

30 M. Mireuta, M. A. Hancock, M. Pollak, J. Biol. Chem. 2011, 286, 16567.

31 T. Yasuoka, T. Takahashi, K. Tanaka, M. Kawashima, Poult. Sci. 2001, 80, 1231

32 G. C. Nicholson, J. M. Moseley, P. M. Sexton, F. A. O. Mendelsohn, T. J. Martin, J. Clin. Investig. 1986, 78, 355.

33 E. Gaucci, D. Raimondo, C. Grillo, L. Cervoni, F. Altieri, G. Nittari, M. Eufemi, S. Chichiarelli, Sci. Rep. 2016, 6, 37957.

34 J. A. Huhtakangas, C. J. Olivera, J. E. Bishop, L. P. Zanello, A. W. Norman, Mol. Endocrinol. 2004, 18, 2660.

35 D.-R. Hwang, E. Hu, J. R. Allen, C. Davis, J. Treanor, S. Miller, H. Chen, B. Shi, T. K. Narayanan, O. Barret, D. Alagille, Z. Yu, M. Slifstein, Nucl. Med. Biol. 2015, 42, 654.
36 M. N. Sillence, J. Hooper, G. H. Zhou, Q. Liu, K. J. Munn, J. Anim. Sci. 2005, 83, 2339.

37 D. A. Sykes, S. J. Charlton, Br. J. Pharmacol. 2012, 165, 2672.

38 S. K. Thangaraj, H. Arola, A. Tullila, T. K. Nevanen, J. Rouvinen, J. Jänis, ACS Omega 2019, 4, 18718.

39 X. Qi, F. Loiseau, W. L. Chan, Y. Yan, Z. Wei, L.-G. Milroy, R. M. Myers, S. V. Ley, R. J. Read, R. W. Carrell, A. Zhou, J. Biol. Chem. 2011, 286, 16163.

40 P. Prapunpoj, L. Leelawatwatana, G. Schreiber, S. J. Richardson, FEBS J. 2006, 273, 4013.

41 N. M. Green, Biochem. J. 1966, 101, 774.

42 N. M. Green, Meth. Enzymol. 1990, 184, 51.

43 Y. Gong, T. C. S. Pace, C. Castillo, C. Bohne, M. A. O'Neill, E. Plettner, Chem. Biol. 2009, 16, 162.

44 P. V. Hornbeck, B. Zhang, B. Murray, J. M. Kornhauser, V. Latham, E. Skrzypek, PhosphoSitePlus, 2014: mutations, PTMs and recalibrations. Nucleic Acids Res. 2015 Jan;43(Database issue):D512-20.

45 S. Kim, J. Chen, T. Cheng, A. Gindulyte, J. He, S. He, Q. Li, B. A. Shoemaker, P. A. Thiessen, B. Yu, L. Zaslavsky, J. Zhang, E. E. Bolton, (2019). PubChem in 2021: new data content and improved web interfaces. Nucleic Acids Res. 2021 49(D1), D1388-D1395. https://doi.org/10.1093/nar/gkaa971

46 ChemIDPlus [Internet]. Bethesda (MD): National Library of Medicine (US), National Center for Biotechnology Information; 2004 - [cited $2021 \mathrm{Feb} 23$ ]. Available from: https://www.ncbi.nlm.nih.gov/ chemidplus/

47 GenomeNet [Internet]. Available from: https://www.genome.jp/

48 https://www.pmda.go.jp/files/000153631.pdf

49 https://www.accessdata.fda.gov/drugsatfda_docs/ label/2005/125084_0030lbl.pdf

50 https://www.accessdata.fda.gov/drugsatfda_docs/ label/2018/761065lbl.pdf

51 https://www.bio-rad-antibodies.com/monoclonal/ mouse-cd117-antibody-2b8-mca1365.html?f=purified

52 https://www.u-proteinexpress.com/sars-cov-2-rbd

53 https://www.rndsystems.com/products/recombinant-sars-cov-2spike-rbd-his-tag-protein-cf_10534-cv

54 https://www.sinobiological.com/recombinant-proteins/sars-covspike-40150-v08b2

55 https://www.u-proteinexpress.com/sars-cov-2-rbd

56 T. Kohno, L.-T. T. Tam, S. R. Stevens, J. S. Louie, J. Investig. Dermatol. Symp. Proc. 2007, 12, 5.

57 https://www.biorxiv.org/content/10.1101/ 2020.03.16.994152v1.full.pdf

58 M. P. Czech, J. Massague, Fed Proc. 1982, 41, 2719.

59 https://www.mybiosource.com/ctr-chicken-elisa-kits/ calcitonin-receptor/747783

60 C. S. Mermelstein, E. R. Martins, D. M. Portilho, M. L. Costa, Cell Tissue Res. 2007, 327, 343.

61 R. Bouillon, F. Schuit, L. Antonio, F. Rastinejad, Front Endocrinol. (Lausanne). 2019, 10, 910.

62 http://www.worthington-biochem.com/AV/

63 D. Xua, S. V. Wegner, Chem. Sci., 2020, 11, 4422. 